\title{
Cine chileno en dos tiempos: para una lectura del desplazamiento del cuadro teatral al encuadre cinematográfico ${ }^{1}$
}

\section{Chilean Cinema in Two Phases: for a Reading About the Displacement From the Theatrical Picture to the Cinematographic Frame}

\section{Marcela Parada Poblete}

Facultad de Arquitectura, Diseño y Estudios Urbanos. Escuela de Diseño, Pontificia Universidad Católica de Chile. Santiago, Chile.

mparadap@uc.cl

\section{Resumen}

Proponemos un cruce de lectura entre la estética del cine silente chileno y sus relaciones con el cine contemporáneo Generación 2000, distinguiendo el desplazamiento del cuadro teatral al encuadre cinematográfico. El cine de los comienzos manifiesta ante todo un interés en sí mismo, y lo cotidiano de sus registros de la realidad opera como pretexto para un recurso que explora sus posibilidades técnicas y estéticas. En el cine contemporáneo se adopta una relación de otro tipo con lo cotidiano, intentando explorar los distintos niveles de sentido en el registro de la realidad y la temporalidad, a la par de una búsqueda del cine como representación e interrogación de mundo.

Palabras clave: Cine chileno, cine silente, cine contemporáneo, documental, representación.

\section{Abstract}

We propose a reading cross between the aesthetics of the Chilean silent cinema and their relationship with contemporary cinema Generation 2000, identifying the movement from the theatrical picture to the cinematographic frame. The early cinema mainly manifests an interest on itself, and the quotidian of their records of reality works as an excuse to a resource that explores their technical and aesthetic possibilities. In contemporary cinema, a different relationship with the quotidian is adopted, trying to explore the different levels of sense in the record of reality and temporality, as well as a search of cinema as representation and interrogation of world.

Keywords: Chilean cinema, Silent films, Contemporary films, Documentary, Representation.

1 Una primera versión de este trabajo fue presentada en el XXX Congreso Internacional de LASA (Latin American Studies Association), realizado en San Francisco, California, en mayo de 2012. 
Examinamos en este estudio el cine silente y el cine contemporáneo chileno, focalizando la atención en la operación visual que recae sobre el recorte de mundo, con su consecuente grado experiencial de representación, en estos dos tiempos de la cinematografía nacional. Advirtiendo, por cierto, la evidencia de la distancia -técnica y temporal- entre los inicios del cine en territorio nacional y el cine de nuestros días, tal elipsis es un riesgo que se ha asumido conscientemente abordar en este estudio, reconociendo que este salto cercano a un siglo posibilita ensayar una lectura a propósito de la reflexión que ha adoptado el cine como técnica de registro y representación, como expresión de posibilidad de comprensión e inscripción de mundo y del sujeto en el mundo.

El análisis lo dividimos en tres partes. Una primera sección se aboca al período del cine silente nacional, del cual revisamos un conjunto de vistas de actualidades sociales y cívicas de la primera década de 1900, las que pueden inscribirse en el naciente carácter documental ${ }^{2}$ y que corresponden -replicando, en primera instancia, el modelo Lumière importado en la época- a la tendencia de registro del cine de los comienzos. Considerando que el espectáculo del cinematógrafo llega a Chile en 1896 y que, con incursiones aisladas en 1897 y 1900, el año 1902 puede ser consignado como el inicio de una producción nacional -escasa o eventualmente sistemática- que se intensifica en 1910 con ocasión del Centenario de la República, para este período hemos seleccionado una serie de cortometrajes del archivo histórico audiovisual chileno que han sido restaurados por la Cineteca Nacional. Entre las vistas que se revisan se hallan: Paseo a Playa Ancha (1903), La exposición de animales (1907), Festejos en el Parque Cousiño (1910), Gran revista militar en el Parque Cousiño (1910) e Inauguración del Palacio de Bellas Artes (1910). El análisis que se desarrolla en esta sección nos lleva a distinguir que el cine de los comienzos manifiesta en la representación una poética de cuadro teatral.

En la segunda parte abordamos el período del cine contemporáneo nacional, para el cual se ha seleccionado un conjunto de realizaciones que hemos considerado como exponentes del nuevo cine nuevo chileno, que se manifiesta en el cine Generación $2000^{3}$, incluyendo puestas en obra de carácter documental y puestas en obra que trabajan en la frontera de la dualidad e indeterminabilidad entre documental y ficción. Sobre esto último, se ha considerado que en todo registro cinematográfico de la realidad -ya sea de carácter documental incipiente, documental o documental

2 La acepción de cine documental, no exenta de discusiones teóricas, la abordamos para este análisis como aquel cine que presenta -con más o menos acuerdos y discrepancias conceptuales- una relación preliminar expresa con la realidad. Para el caso del cine silente, corresponderá por tanto a esta designación, el registro de situaciones que -en su momento- equivalen a actualidades cuyo carácter de actualidad, pese a desintegrarse en el propio registro, apuntan a fragmentos de el mundo (concordando aquí con la distinción de Nichols) que una vez algunos, otros, aquellos, habitaron (y también posaron) para el registro cinematográfico y su reproducción.

3 Un cine doblemente nuevo, considerando que la denominación de "Nuevo cine chileno" se asocia históricamente al Festival de cine de Viña del Mar de 1969. Para una revisión de ello ver: Parada, Marcela. "Los estudios sobre cine en Chile: una visión panorámica 1960-2009". Revista Razón y Palabra 77 (2011). 
atravesado por la ficción- es posible hallar un recorte de mundo que, entre el registro y el discurso, devela una actitud y un prisma particular de acercar el propio mundo en la puesta en obra de una representación ${ }^{4}$. Para este período, nos situamos en una parcela particular del cine contemporáneo, aquel que se desprende -en la época- del cine que y como se venía haciendo, y que trabaja en la exploración de los distintos niveles de sentido alojados en la temporalidad del registro y cifrados en la escala cotidiana de percepción e interrogación de mundo. Revisamos en esta sección un conjunto de realizaciones entre las que se hallan: Ningún lugar en ninguna parte (2004), Obreras saliendo de la fábrica (2005), Rabia (2006), El pejesapo (2007), Noticias (2009) y Manuel de Ribera (2010). El análisis nos conduce a la noción de la poética del encuadre cinematográfico.

En la tercera parte se desarrolla un cruce de lectura entre ambos modelos de representación, centrándonos en la operación visual que recae sobre el recorte de mundo en estos dos tiempos de la cinematografía nacional, y cuyos alcances se reconocen atravesados por la emergencia y desplazamiento del cuadro-teatral al encuadre-cinematográfico, con las implicancias artísticas y de representación de mundo que ello convoca.

\section{La poética del cuadro teatral en el cine silente nacional}

Et les hommes ont vu que le monde était là, un monde encore presque sans histoire, mais un monde qui raconte ${ }^{5}$ Godard, en: Histoire(s) du Cinéma (1998)

El cine llega a Chile el 25 de agosto de 1896, cuando a ocho meses del histórico estreno en París, en el teatro Unión Central de Santiago se exhibe el espectáculo del cinematógrafo Lumière que por entonces da la vuelta al mundo. El programa contempla la

4 En ello, discrepamos de Campo cuando refiriéndose a las vistas Lumière menciona que en ellas "no está el comienzo del discurso documental, sino del registro de lo real." Y agrega "Como destaca el crítico argentino Emilio Bernini, en los cortos de Lumière se puede ver más el asombro y el encanto por el movimiento antes que la invención o el descubrimiento de otro" (277). Por cierto que la fascinación y extrañeza ha de haber impregnado al público asistente al espectáculo de las vistas en movimiento de entonces; pero consideramos que, tanto del lado del registro como del lado del espectador, ya sea por instinto y/o inclinación de sentido narrativo, el ya registro de lo real -o más precisamente de la realidad, para evitar nuevas discrepancias conceptuales entre, digamos, lo real y la realidad-, puede ser leído, incluso en aquella época temprana de las vistas Lumière, como un discurso documental, aunque sea en su modo más germinal. Es que, ¿es posible descartar la intención discursiva que subyace, aún cuando sea instintivamente, en toda expresión humana?. Será, acaso, que esa misma inclinación a hallar un sentido narrativo es la que nos precede, también a nosotros, en esta aseveración a propósito del documental germinal. Insistencias, ciertamente, de la propia subjetividad.

5 "Y los hombres vieron que el mundo estaba allí, un mundo aún casi sin historia, pero un mundo que cuenta" (La traducción es mía). 
proyección de las vistas La comida del bebé, Los jugadores de cartas y La llegada del tren, entre otras; ante lo que el diario El Ferrocarril, al día siguiente del estreno, consigna: A primera vista se diría que aquello es una pantalla en que se reproducen imágenes o personajes, pero no bien empieza a funcionar, se apodera del espectador la más extraña sensación de luz, movimiento y vida, que le transporta por obra de magia a las rápidas, interesantes y variadas escenas de la vida real [...] La ilusión que produce el cinematógrafo es perfecta, es en realidad la prolongación de la vida. (cit. en Jara, Cine mudo chileno 16).

Junto con notificar y reseñar el hecho -histórico, ciertamente- de la llegada de estas vistas animadas al país, este comentario constituye la primera aproximación crítica nacional al espectáculo cinematográfico y al efecto de realidad que éste convoca. Para el caso, la noción de reproducción de personajes, precedida por la técnica fotográfica, aparece desde este momento inaugural asociada no sólo a la sensación de luz y a la impresión de movimiento, si no que con ello - o como consecuencia de ello- a la vida, a la impresión de vida. Fijemos, por tanto, que en estas vistas tempranas la impresión del movimiento de los personajes, dada por la novedad de la facultad técnica de posibilitar el registro imprimible de fotogramas sucesivos y su posterior proyección, desencadena la impresión de vida, dada por la reproducción -y reactualización- de una acción en cuadro. Impresión, pues, de doble vía: como técnica y como percepción; sistema en el que técnica de registro de mundo y representación de mundo comienzan, inauguralmente, a alinearse ${ }^{6}$. Impresión de movimiento e impresión de vida. Detengámonos, aún, brevemente en ello... "La ilusión que produce el cinematógrafo es perfecta. Es en realidad la prolongación de la vida." En esta acepción de "prolongación de la vida" distinguimos, así mismo, que subyace la noción -ciertamente incipiente- respecto de la técnica, su condición de registro y la imagen de mundo en la representación. Es más, el texto refiere a "la ilusión que produce el cinematógrafo", y en ello emerge -de forma notable y ya en la época-, la impresión de una condición imaginaria, espectral, que nos sitúa en el terreno de la reproductibilidad técnica de mundo, de la posible representación de mundo. Por su parte, en cuanto a las situaciones de registro, en estas vistas Lumière el espectador asiste a la reproducción de episodios aislados, escenas -en rigor- en las que la acción que se expone se desarrolla con unidad de tiempo y lugar; con la salvedad de que estas escenas Lumière son únicas, no están asociadas a un sistema de articulación significante de fragmentos de realidad -como entendemos con posterioridad a esta época el Cine toda vez que ha incorporado el montaje- y en

6 Alineación, en cualquier caso, que siempre será ilusoria, incluso en déficit o en falta. ¿Cómo podría acontecer la alineación estricta entre registro de mundo y su representación si no es a través de una condición aparente y figurada de concordancia, si no es a través de un imaginario de representación? "Sistema en el que técnica de registro de mundo y representación de mundo comienzan, inauguralmente, a alinearse." Alineación que considera la noción del cine como asíntota de la realidad; iluminadora metáfora geométrica enunciada en su momento por Bazin: el cine como una línea recta prolongada indefinidamente, acercándose a la curva de la realidad, dependiendo de ésta y, con todo, sin alcanzarla jamás. 
cuanto significan lo hacen por sí mismas, de modo particularmente unitario. En otras palabras, estamos ante escenas-isla, segmentos cercanos al minuto de acciones - por el momento digamos cotidianas, incluso hasta mínimas-, en las que el común denominador lo constituye precisamente la acción que despliegan los sujetos en cuadro. Los títulos, por su parte, reseñan literalmente el acto registrado y reproducido.

Respecto a la producción de vistas nacionales, la primera incursión cinematográfica de la que se tiene noticia data de mayo de 1897, cuando a nueve meses del estreno de las vistas Lumière en el país, el Salón de la Filarmónica de Iquique exhibe una serie de vistas realizadas por Luis Oddó, fotógrafo radicado en la misma ciudad. Entre las vistas proyectadas en aquel Salón, entre mayo y junio de ese año, se hallan: El desfile en honor del Brasil, Una cueca en Cavancha, La llegada de un tren de pasajeros a la estación de Iquique, Bomba Tarapacá No 7 y Grupos de gananciosos en la partida Football entre caballeros de Iquique y de la Pampa. (Jara, Una breve mirada al cine mudo 176). Tal temprana muestra será espasmódicamente seguida por la del 10 de agosto de 1900, cuando el Teatro Olimpo de Santiago, dentro del programa de vistas extranjeras, intercala la exhibición de Las carreras de Viña del Mar, registro que refiere al espectáculo de las carreras de caballos en la costa central de Chile (Vega 45); proyección apenas mencionada por la prensa de la época y, como consecuencia, de limitado rastreo histórico en la actualidad. A continuación, el 26 de mayo de 1902, se estrena en el teatro Odeón de Valparaíso Ejercicio jeneral del Cuerpo de Bombe$\operatorname{ros}^{7}$, cortometraje que bordea los dos minutos en el que se registra-y reproduce- el ejercicio que ha realizado -en abril de ese mismo año- el Cuerpo de Bomberos en la plaza pública de la ciudad. La película estaba compuesta por varias tomas: Desfile de las compañías por la Plaza Aníbal Pinto, Competencia de grifos, Ejercicios de escaleraLa pirámide y Bautizo de la II Compañía. Seguidamente, el cine silente chileno de los primeros años se aboca a capturar escenas de la vida cotidiana, focalizadas - para el caso- en las actualidades cívicas o sociales de turno. Paseos y encuentros de la élite, actos públicos y ceremonias oficiales son los primeros motivos de registro que atraen a las cámaras y al público a las salas de proyección.

De ello, es claro reconocer que el cine chileno de los comienzos transcribe -en primera instancia y a su modo, tal y como ocurre en la mayoría de los países-, el modelo Lumière importado en la época ${ }^{8}$. Las vistas nos sitúan ante lo que hemos llamado escenas-isla; segmentos breves, de situaciones aisladas, y en donde la vida de "otros" se desenvuelve en panoramas de paisajes en actividad. En cualquier caso,

7 También conocida como Ejercicio General de Bombas.

8 Consideremos, por ejemplo, que La llegada del tren a la estación (Lumière, 1895) puede ser homologada a La llegada de un tren de pasajeros a la estación de Iquique (Oddó, 1897), así como Paseo de los Ostrichers en el jardín botánico de París (Lumière, 1895) encuentra su equivalente en La exposición de animales (Real, 1907), o bien, La llegada de los congresistas a Neuville Sur-Saone (Lumière, 1895) encuentra correspondencias en gran parte de las vistas nacionales realizadas en el marco del acontecimiento de "el San Martín en aguas chilenas" (1903) y en la toma de vistas de "las llegadas" a suelo nacional de visitas ilustres con ocasión de las celebraciones del Centenario (1910). 
este "otros" es, ciertamente, un decir. Las situaciones de registro convocan en el cuadro-encuadre de los primeros tiempos a la clase acomodada con su respectivo despliegue protagónico. Cuestión que resulta evidente en una nota del 14 de abril de 1903 del Diario Las Últimas Noticias, que refiere al espectáculo ofrecido por el teatro Variedades de Santiago: "La sala de este simpático teatrito se ha visto noche a noche llena de espectadores, pertenecientes a nuestra mejor sociedad, deseosa de ver a sus amigos y amigas sobre la blanca tela en que se reflejan las vistas. Allí se ven desfilar a los jóvenes más conocidos de nuestro mundo social.” (cit. en Godoy 15).

Ahora bien, este "otros" es siempre multitud, abundante en personas-personaje que acuden a la zona de registro de la cámara donde el cuadro se organizaba según una composición que podríamos denominar teatral. En esta alusión a teatralidad nos referimos al modo de representación teatral más convencional, en donde la forma rectangular del escenario convoca un cuadro dramático que confiere valor a la acción representada en los límites interiores del rectángulo, privilegiando esta área como centro espacial y foco dramático. En esta línea, el movimiento de concentración espacial de acción que distinguimos convoca el cine de los comienzos puede ser desplazado al concepto de composición teatral de modelo tradicional ${ }^{9}$.

Por su parte, la escena-isla que constituye cada vista queda marcada -como en el teatro de lenguaje convencional- por la entrada y salida de los personajes a cuadro, sujetos-objeto de registro que -para el caso- actúan en bloque, en multitud; como puede apreciarse en Paseo a Playa Ancha (1903) y en La Exposición de animales (1907) ${ }^{10}$. En la primera vista, el motivo de registro es el almuerzo al aire libre ofrecido para 150 comensales por la Empresa de A. Massonier ${ }^{11}$; reunión que incluye un pie de cueca de

9 A propósito del espacio escénico y la composición del cuadro teatral, nos apoyamos con el texto La dramaturgia del espacio, de Ramón Griffero. Si bien su trabajo se aboca a proponer "un estudio sobre el arte escénico a partir de la práctica y una reflexión sobre su formato: el espacio" (17), para lo cual desarrolla todo un sistema de ejercicios prácticos actorales dirigidos a experimentar y comprender las posibilidades de composición dentro del rectángulo que van generando una narrativa espacial particular; en el primer capítulo, el autor refiere a la forma geométrica del formato espacial "que hemos adoptado, históricamente, para plasmar nuestras expresiones artístico-culturales, ya que la geometría del formato ha sido una plantilla para las narrativas visuales artísticas que hemos desarrollado" (55). Sobre ello, distingue la primacía de la forma rectangular, señalando que a propósito del espacio escénico y el edificio teatral que alberga en distintas épocas las distintas representaciones, todos cobijan el rectángulo como el centro del espacio teatral (62). Junto a lo anterior, en el capítulo 2, el primer ejercicio práctico que propone para los actores es solicitarles, desde el centro del escenario, que se dispongan en los contornos de éste (en los límites exteriores del rectángulo), dejando libre el área frontal. Luego se les indica que entren al espacio y enuncien su nombre. Lo que ocurre entonces, en la práctica actoral, es que la mayoría de los participantes tiende a detenerse en el centro. Claro está que desde allí se profirieron las instrucciones. Con todo, señala Griffero: "Esto sucede ya que en primera instancia se aplican las conductas cotidianas del cuerpo en un escenario, reiteradas sin mayor reflexión sobre una escena: la tendencia es que cuando el intérprete sube a decir su nombre, no lo dirá desde un rincón, sino que se sitúa en el centro, como quien va a recibir un diploma o posar para una foto" (75). Desde aquí, desprendemos en parte la hegemonía del cuadro-escenario teatral y el centro como zona de parlamento y foco privilegiado de acción, que se manifiesta en la disposición teatral del cine de los comienzos.

10 La exposición de animales también es conocida como Vista de la Quinta Normal. Para esta vista -así como para otros registros cinematográficos silentes que retomamos en este trabajo- contamos hoy sólo con fragmentos que han resistido al paso del tiempo siendo restaurados por la Cineteca Nacional. De modo que el ensayo de lectura que elaboramos es, a su vez, basado en tales fragmentos.

11 Operador de cámara francés formado profesionalmente en la empresa Lumière. A mediados de 1902 se radica en Chile e instala la Empresa Massonier Ca. -productora cinematográfica- y el Biógrafo Lumière. 
conjunto animado por cantoras, palmas y guitarras, una pelea simulada y una gran mesa de reunión ante la que se brinda para la cámara y se hace pasar un cordero asado al palo. Este almuerzo se lleva a cabo el 8 de enero y la vista se estrena el día 16. En la segunda vista, el motivo de registro es - como el título lo señala- la exposición de animales realizada anualmente en octubre de ese año en la Quinta Normal. Como señala Alicia Vega, se trata de un acontecimiento "de extraordinaria relevancia comercial, científica y social, organizado por la Sociedad Nacional de Agricultura y la Sociedad de Fomento de Razas Caballeras" (Itinerario del cine documental chileno 52). La vista se estrena el 13 de noviembre de 1907. Llama la atención que este registro, al menos en el fragmento que ha sido restaurado por la Cineteca Nacional, se centra en el desfile del público asistente a la exposición. Los animales, motivo de reunión, y las vistas generales del lugar quedan ausentes, siendo remitidos apenas por el título de la vista.

Fijemos que en ambas realizaciones, el retrato social animado es primordial. La zona de registro actúa como un imán, atrayendo a un número francamente indeterminado de personas que transitan en los límites interiores del cuadro. En Paseo a Playa Ancha, el movimiento de atracción es central. La distintas acciones de los comensales desfilan secuencialmente al interior de un plano de conjunto fijo, donde el centro del cuadro es el foco de registro, de acción y atención. En el transcurso, el ir y venir de los danzantes de cueca deja entrever, en el fondo de la composición, la mesa que recibirá el banquete, así como un cartel, hacia la derecha del cuadro, que fija el lugar y la fecha: "Playa Ancha. Valparaíso. Enero 8 de 19..." Asistimos a una suerte de estampa pictórica-teatral en movimiento de concentración central, y en la que se ha cuidado que la misma vista exhiba sus coordenadas de sujeción espacialtemporal con una leyenda que ha sido incorporada en la misma composición y que actúa a la manera de un pié de foto de la estampa en movimiento. Junto a lo anterior, cabe destacar que en los últimos segundos de la vista se percibe un corte de registro. Una interrupción mínima, un tiempo que se suelda prontamente. Para cuando se retoma la vista animada, la gran mesa del banquete ha sido desplazada de su posición original para quedar al centro de la zona pictórica-teatral. En términos compositivos, la disposición de la mesa se extiende hacia el fondo del cuadro y la rodea el numeroso grupo de comensales. Es el brindis hacia cámara y el final de la vista, en donde todo ha sido dispuesto para corresponder al retrato social animado del conjunto en un riguroso plano, precisamente de conjunto, sobre la multitud. En el caso de $\mathrm{La}$ exposición de animales, el movimiento de atracción de los sujetos-objeto de registro sobre el cuadro es horizontal. Sobre un plano de conjunto fijo, el desfile del público asistente a la exposición atraviesa la zona de registro de izquierda a derecha. Hacia el fondo de la composición, es posible vislumbrar algunos carruajes tirados por caballos. Con todo, la atención se concentra en la hilera de personas-personaje que cruza el cuadro. Hombres, mujeres, niños y niñas ataviados elegantemente. Todos desfilan con refinamiento al interior del cuadro, ninguno corre y ninguno se apresura en tomar su turno. Cada cual toma su tiempo para concurrir al espacio escénico del retrato 
social en movimiento. Transcurrido cerca de un minuto, la vista presenta un corte explícito en el registro. El cuadro se retoma con un plano que se ha cerrado sobre un conjunto más cercano: un automóvil repleto de elegantes varones y damas cruza la composición. Seguidamente, la secuencia se articula con otras de carácter similar. La multitud se agrupa y transita alrededor de los carruajes y el gentío continúa desfilando en el evento social. Un joven que transita en medio de la aglomeración se quita su sombrero y saluda a cámara antes de abandonar el cuadro. La vista finaliza con una secuencia en la misma línea de la inicial. Plano de conjunto del desfile social asistente al evento, a una distancia que abandona la pseudo proximidad sobre las personaspersonaje, reafirmando el retrato pictórico en movimiento de la multitud.

Con lo anterior, es claro que Paseo a Playa Ancha y La exposición de animales manifiestan un movimiento de atracción sobre el cuadro. En el primer caso, y como hemos referido, el movimiento de atracción es central, en el segundo, es horizontal. Pero en ambos, la zona de registro actúa como un polo magnético que tiene la facultad de congregar lo múltiple en un panorama de paisaje en actividad, registrando y perpetuando un instante de la multitud en movimiento; cuestión que va de la mano con el efecto de realidad que provoca el Cinematógrafo en los espectadores de entonces. El movimiento, claro está, adopta el nudo de la cuestión.

Con ello, en el cine de los comienzos estamos ante una variante del efecto de choque acuñado por Walter Benjamin (Discursos interrumpidos I 51) en su momento. No es necesario incorporar el cambio radical de escenas o de planos, los cortes determinantes que incorpora el montaje y que, por obra del lenguaje cinematográfico simulan -o proporcionan la ilusión de-continuidad espacial y temporal en una estructura fragmentaria. El choque inaugural es producto del movimiento y de la posibilidad de encapsular el tiempo. Es producto de la reproductibilidad técnica y de la fascinación por la inscripción del movimiento, por la posibilidad que otorga la técnica de contemplación de un mundo en acto que, pese a presentarse como la representación de una naturaleza en déficit ${ }^{12}$, devuelve la impresión de vida. Como señala Doane, en su estudio de la emergencia del tiempo cinemático: “Gran parte de la retórica que acompaña la acogida de las primeras películas es una celebración de la capacidad que posee el cine para representar el movimiento. Mientras que la fotografía podía fijar un momento, el cine hacía archivable la propia duración" (La emergencia del tiempo cinemático 46).

Hasta aquí estamos con el efecto de choque del lado del espectador. Del lado de la zona de registro, en tanto, si la locación única y el plano sostenido sobre la acción en cuadro, acusan un sistema teatral, el movimiento de congregación que allí opera -pese a corresponder a la época muda- instaura una suerte de "zona de parlamento" que intensifica dicho sistema convocando al torrente de personas-personaje al interior

12 Para el caso, déficit técnico: imagen en blanco y negro, desprovista de sonido ambiente y proyectada a una velocidad de doce cuadros por segundo. Y déficit, ciertamente, de presentación. La presentación imposible que subyace en toda representación. (Insistencias, una vez más, en el final de esta nota de la propia subjetividad). 
de sus límites. Para esta noción de zona de parlamento retomamos aquí la alusión al teatro tradicional, donde la convención espacial de la forma rectangular del escenario determina, a su vez, una zona de discurso, lugar desde donde son proferidos los diálogos hacia el público-observador que se halla en frente de la escena. Ahora bien, en el caso del cine de los comienzos, aunque resulte obvio que la novedad de la técnica provoca tal movimiento de concentración, lo que nos interesa destacar aquí es el espesor narrativo-técnico que adopta y proyecta esta composición.

A la manera del modelo Lumière, el cine silente chileno sostiene sobre el paisaje en actividad un Plano de Conjunto de la situación grupal ${ }^{13}$. A veces el plano es abierto, otras es cerrado, o bien, en el caso chileno, opera con la combinación de ambos, como puede apreciarse en Festejos en el Parque Cousiño o en Gran Revista Militar en el Parque Cousiño (1910) que alternan en cada vista ambas distancias de observación. Tales registros, realizados con ocasión de las celebraciones y actos oficiales del Centenario de la República presentan, aún con la variante técnico-compositiva que incorpora distintos planos de conjunto sobre la acción-motivo de registro, un espesor teatral; dado por el movimiento de concentración de la acción en la zona de parlamento que es el cuadro escénico. A modo de referencia, Festejos en el Parque Cousiño inicia con un plano medio de conjunto sobre un grupo considerable de hombres y niños que se agrupa en la zona de registro, en donde cada cual disputa con los demás-levemente, en clima de festejo- el protagonismo al centro del cuadro. Algunos hombres beben vino directamente desde una garrafa que pasa de mano en mano, alternándose la primacía del protagonismo de la acción. Los niños, entretanto, se aglomeran con el conjunto festivo, miran a cámara y saludan desde el centro del cuadro. Un leve corte en el registro nos sitúa en un nuevo plano de conjunto que ahora recae sobre un grupo de hombres montados a caballo, donde cada cual espolea a su animal para pasar, alternadamente, al centro de la zona de parlamento. El siguiente corte nos deja con un plano de conjunto a mayor distancia sobre un grupo de hombres, mujeres, niños y niñas que rodea y aviva con palmas y guitarra a una pareja que, al centro de la zona de registro, baila un pie de cueca. El público se ubica en semicírculo alrededor de los danzantes, dejando libre la zona de la cámara y alternando su atención entre la pareja que baila y aquella cámara que los fotografía, a todos, en movimiento. El final de la vista, es el final del baile. El foco continúa siendo la pareja de la cueca, siempre al centro del cuadro, mientras el público todavía se agolpa para perseverar en la zona de parlamento.

En el caso de Gran Revista Militar en el Parque Cousiño, la vista se inicia con un breve plano picado sobre un automóvil en el que, se diría, llegan algunas autoridades al desfile: un grupo de caballeros con tenida formal, a los que sólo podemos apreciar en plano cenital y de espaldas. Tras algunos segundos, el siguiente ángulo de cámara es normal, en un plano distante del desfile militar y en el que puede apre-

13 Pensemos, por ejemplo, en las vistas Lumière de Obreros saliendo de la fábrica (1895), Paseo de los Ostrichers en el jardín botánico de París (1895), o en Batalla de las bolas de nieve (1896). 
ciarse la marcha oficial de conjunto. En esta vista, permanecemos preferentemente allí, en la acción del desfile. Asistimos a un despliegue visual de un grupo incontable de hombres que atraviesan alineados el cuadro en sentido horizontal de izquierda a derecha. En esta vista así como en las otras que hemos referido, la gran cantidad de personas-personaje que posan y desfilan en el encuadre es siempre numerosa; cuestión que se multiplica, incluso, por obra del tiempo de auscultación. Dicho de otro modo, el plano sostenido sobre la aglomeración, sobre el gentío indeterminado, funciona como un multiplicador temporal y representacional. ¿Cuántas personas desfilan allí? ¿Quiénes desfilan? ¿Desfilan algunos más de una vez al interior del cuadro? ¿Cuánto tiempo, digamos "efectivo", digamos "matemático", transcurre en este lapso de tiempo registrado sobre una multitud? La respuesta específica no es, del todo, relevante. La pregunta por cuántos sujetos y por cuánto tiempo lo que hace es trasladarnos al plano de la representación, en donde el minuto matemático-técnico se extiende por obra del mecanismo que dirige la mirada y fija la atención sobre la masa en movimiento. Lo determinante, pues, es el plano sostenido sobre una multitud, el plano-masa, más aún: el plano sostenido sobre un rostro-masa.

Sobre ello distinguimos la vista Inauguración del Palacio de Bellas Artes (1910), en la cual el motivo es, claro está, la inauguración del recinto, ceremonia que se llevó a cabo el 21 de septiembre en el marco de las actividades del Centenario ${ }^{14}$. La vista total queda articulada en tres segmentos: multitud, fachada y multitud. En ello destaca, en primera instancia, la incorporación del micro segmento central, con un breve panorama del frontis de la construcción. La referencia espacial funciona como un insert y puede apreciarse que fue registrada con anterioridad al día del evento. La fachada está despejada y la vemos en Plano General. El cuadro de la solemne construcción es animado por algunos jardineros que rondan el perímetro. La toma presenta un corte. Seguidamente es el detalle de banderas flameando al viento, un leve paneo desde las banderas y sobre la fachada para luego continuar con un leve contrapicado hacia la cúspide del Palacio. Fin del insert. Reconociendo la eficacia de este inserción espacial referencial -cuestión que distinguíamos ausente en la vista La exposición de animales- nos detenemos en los segmentos de multitud de inicio y final que dan forma a la vista de “inauguración”. En ambos, el gentío en el exterior del Palacio es determinante. Evidentemente, estamos ante el registro de una inauguración; no obstante la aglomeración humana no es exclusiva de esta vista, si no que, como hemos revisado, constituye el sistema representacional del cine silente. Para el primer segmento de multitud, el cuadro-encuadre presenta un movimiento interno en sentido horizontal. En el segundo, el movimiento es externo, la cámara se mueve sobre el conjunto de personas que asiste al discurso inaugural proferido en el podio. Ahora bien, en esta vista el plano sostenido sobre la aglomeración presenta un indicio de reflexión crucial: la multitud concurre a su disposición teatral y coral, no sólo llenando la zona de registro, si no

14 La vista se estrena el 26 de septiembre de 1910. 
que, para el caso, este trayecto al interior del cuadro-encuadre incorpora de forma determinante el retardo. En el tránsito, el gentío se demora, particularmente, al pasar por la zona de registro. Algunos sectores del rostro-masa miran detenidamente hacia cámara, se quedan allí unos instantes, insisten en permanecer al interior de la demarcación espacial del cuadro antes de continuar con el tránsito. Y en ello, producto de este retardo, la zona de parlamento de la multitud teatral se intensifica.

Así las cosas, en esta dominante de exposición -que es, a la vez, una dominante de representación- el encuadre emerge como límite de exhibición y auscultación, único centro de gravedad narrativa y límite privilegiado para la trascendencia espectacular de la multitud. Eso, en primer término. En segundo término, es posible vislumbrar que, producto del retardo propiciado por el gentío al interior del cuadro así como del lado del espectador, la mirada del rostro-masa se proyecta, germinalmente, hacia un sitio que va más allá del ojo del aparato de registro, más allá -incluso-del espectador, hacia una dimensión otra. Es, quizá, el destello del extrañamiento. Un extrañamiento incipiente, volcado sobre el mecanismo de registro y reproducción, sobre el aparato que perpetúa el movimiento, la sensación de vida. Un extrañamiento primero que más tarde, en el cine contemporáneo, se volverá de lleno sobre el mundo circundante.

Sobre estas distinciones del cine silente y como se ha referido en las primeras líneas de este trabajo, hemos escogido el riesgo de abordar la elipsis extrema. El quiebre narrativo-técnico entre dos siglos y la reflexión sobre el espesor representacional que de ello emerge.

\section{La poética del encuadre cinematográfico en el cine contemporáneo chileno}

Acá siempre andan inventando historias de hombres solos, de personas solas... Porque está lleno de lugares vacíos... Historias tontas... así es no más.

Voz off al inicio del film Manuel de Ribera (Carrera y Murray, 2010)

El cine chileno Generación $2000^{15}$ sobre el cual focalizamos la atención convive temporalmente -por cierto- con ese otro cine chileno contemporáneo de carácter

15 Parte de esta reflexión a propósito de la categoría Cine Generación 2000 fue presentada en el XV Encuentro de SOCINE - Sociedad Brasilera de Estudios de Cine y Audiovisual-, realizado en Río de Janeiro, Brasil, del 20 al 24 de septiembre de 2011. Ponencia presentada: Cine chileno Generación 2000: la renovación narrativo-técnica; y en la sesión Cine chileno contemporáneo, organizada por el Centro Brasilero de Estudios de América Latina. Fundación Memorial de América Latina, Sao Paulo, Brasil, 24 de septiembre de 2011. Ponencia presentada: Estética del Nuevo Cine Chileno Generación 2000. 
convencional -como podría ser el caso de Machuca (Wood, 2004), Fuga (Larraín, 2006), Dawson. Isla 10 (Littin, 2009) o La Nana (Silva, 2009)-. Un cine de factura tradicional y de tradición cinematográfica que, independiente del tema que aborde y de la destreza con que lo emprenda, se inclina a trabajar narrativa y técnicamente puestas en escena de modelo clásico; categoría que, como distingue Lauro Zavala, al respetar las convenciones en el empleo de los recursos audiovisuales y debido precisamente- a la tradición, presenta una naturaleza didáctica que promueve una lógica de fácil reconocimiento por parte del espectador sobre el sentido narrativo de la obra. El cine contemporáneo que hemos identificado como Generación 2000, en tanto, se desmarca de un modelo, digamos, "aséptico" de representación. Contraviene los formalismos, como alguna vez lo hizo, a su modo, el cine nacional de los 60 y 70. Se desprende, en definitiva, del modelo narrativo-técnico tradicional o convencional, complejizando particularmente el tema de la mirada en y sobre la representación. Como señala José Luis Sepúlveda, realizador de El Pejesapo (2007): “No queríamos estar cercanos a la idea del cine que se está haciendo, porque pensamos que es demasiado elitista, es demasiado conservadora, y podríamos hablar una hora sobre el concepto de conservador, pero siento que ha proclamado una idea de prepotencia en nuestras imágenes, en la imagen de la persona común y corriente"16. O como señala Iván Osnovikoff, realizador junto a Bettina Perut del film Noticias (2009): "No utilizamos el cine como una herramienta pedagógica para exponer discursos ya elaborados en otros formatos, si no ser una herramienta de investigación de la realidad y, por lo tanto, si es_una herramienta de investigación lo que tiene que ir generando es preguntas y respuestas inacabadas que generen nuevas visiones del mundo" ${ }^{17}$. El cine que identificamos como Generación 2000, en otras palabras, es un cine dirigido por excéntricos y astutos, como refiere Carlos Flores: "Jóvenes realizadores que han logrado desarrollar una incipiente estética que reemplaza y combina la influencia del cine clásico por la introspección, la hibridación y la invención (Excéntricos y astutos 12). En este sentido, los filmes que adhieren a la categoría cine contemporáneo que abordamos para este estudio, no lo hacen sólo por una cuestión de época y actualidad, sino que corresponden a aquellos filmes que adoptan una estrategia discursiva operacional que viene a desestabilizar la imagen representacional de mundo y en ello se instalan como marca de un nuevo modelo en la contemporaneidad. Cuestión que establece, por cierto, un campo de análisis crítico sobre la imagen representacional del propio mundo contemporáneo que habitamos a diario.

Referimos, a continuación, las características que distinguimos como transversales en este cine Generación 2000, fijando la atención en cinco aspectos: operativos, materiales, argumentales, representacionales y formales. 
En términos operativos y de producción, este cine restringe en muchos casos recursos, actores, equipos de producción, guiones y hasta tiempos de grabación; cuestión que puede ser leída como un sistema en el que la limitación -decisión de limitación, claro está-, es capitalizada estéticamente en la exploración y reflexión de las operaciones materiales comprendidas en la representación audiovisual de mundo. Y es que este cine es riesgo y es método. A modo de referencia, en Manuel de Ribera (Carrera y Murray 2010), el equipo se traslada con el personaje protagónico al archipiélago de Calbuco y se adentra en la grabación del film con el guion tan solo del primer día: Un hombre que ha heredado una isla deshabitada y que se dirige allí a tomar posesión del lugar. Sin familia, sin amigos, sin trabajo, ni en un lado del mar ni en el otro, los esfuerzos del sujeto se focalizan en la voluntad de fundación territorial. Sobre esta base argumental y como señalan los directores: "Diseñamos un método de trabajo en el cual lo único que conocíamos de la historia en el momento de ir a rodar la película era simplemente esas primeras líneas [...] Y tal como Manuel [el protagonista] va a la conquista de este lugar, de cierta manera también nosotros enfrentarnos a la conquista de este relato"18 (Canal elbuentiempo, web). En la isla, Manuel-personaje y Manuel-persona se entremezcla e interactúa, día a día, con personas del mundo "real" y cada noche el guion para el nuevo día consigna estos encuentros. En palabras de los directores:

La idea de elaborar un proceso creativo de ese tipo era porque era también enfrentarse a qué iba ocurriendo al ir incluyendo los elementos que surgían en el camino, es decir, los elementos humanos de las personas que vivían realmente en ese lugar, también los materiales geográficos del espacio y también estos elementos ficcionales que eran los pocos actores que teníamos. Y de esa manera ir cada día escribiendo, cada día rodando e ir descubriendo este relato en el camino (Ibíd.).

En el caso de El pejesapo (Sepúlveda, 2007), el protagonista es Héctor Morales, ex reo de la cárcel Colina I en donde participó en distintos montajes teatrales estando detenido. Una vez que Héctor sale de la cárcel, el director lo recluta para su película. El film trabaja sobre la base narrativa de un sujeto que ha decidido suicidarse lanzándose al río y que el propio canal ha devuelto a la superficie reintegrándolo a su condición de arrojado sobre la tierra. Desde allí se inicia el viaje, en el que el protagonista se desplaza desde la periferia al centro de la ciudad. Como señala el director:

Ese era el viaje, nada más. No teníamos ninguna otra pretensión más que esa.

[...] Creo que nosotros estábamos trabajando desde la perspectiva del error, de la espontaneidad que va sucediendo a través de los conceptos, y pienso que esa misma espontaneidad y esa frescura y ese mismo error están dentro de lo que no hace la gran mayoría, que ocupa esa estética mucho más clásica, con mayor presupuesto, sino que el planteamiento estético de nosotros era estar ahí, vivir ahí y respirar (Chile independiente, web).

18 Canal elbuentiempo: http://www.youtube.com/watch?v=C5_j-3h2k40. 
Así las cosas, estamos ante realizaciones que se dan a poner en forma una experiencia sobre la realidad, filmes que articulan una forma de pensamiento y aprehensión sobre lo real, y que no simplemente conforman un modelo de producción-registroreproducción de la realidad.

En términos materiales, este cine incorpora registros de diversa índole: cine, digital 8, miniDV, VHS, HDV, incluso en una misma realización -como podría ser el caso de El pejesapo, 31 de abril (Cubillos, 2009) y Naomi Campbel (Donoso y Videla, 2013)-, contraviniendo una puesta en forma de mirada materialmente uniforme, producto de lo cual, la recepción por parte del espectador adquiere una condición de choque óptico-material.

En términos argumentales generales -aunque conscientes de que todo intento de clasificación es arbitrario y limitativo-, sería posible mencionar que este cine nos sitúa ante historias de trazos nimios y personajes comunes. Un cine que pone en obra las grandes proezas de las pequeñas gentes: un joven extranjero que quiere hacer un cortometraje acerca de Santiago de Chile y cámara en mano recorre la ciudad entrevistando a distintas personas (Lavanderos, Y las vacas vuelan 2003); una joven secretaria, desempleada, que busca trabajo y la cámara la sigue en sus múltiples entrevistas infructuosas (Cárdenas, Rabia 2006); un adolescente que tras la muerte de su hermano mayor en el extranjero decide recopilar los videos caseros que éste grabara junto a sus amigos a lo largo de su vida y realizar con ello un documental entrevistando a los "protagonistas VHS" así como a sus padres (31 de abril); las trabajadoras de una fábrica textil, cuyo día a día transcurre en silencio, entre el sonido de las maquinarias y la automatización, y que una tarde, al finalizar la jornada de trabajo, van a la playa y se sientan en la arena ante el mar. (Torres Leiva, Obreras saliendo de la fábrica 2005). Un cine que pone en obra proezas individuales, íntimas, personales, arraigadas en la identidad, en el "poder ser" del sujeto, en la pertenencia, en la inscripción. En la posibilidad de inscripción, posibilidad de sentido. Como enuncia en alguna escena la protagonista inicial del film Mitómana:

Estoy aquí porque soy igual que tú, porque soy una persona más. Y así como tú, quisiera estar contenta. Quisiera bailar, cantar. Pero no me es posible. ¿Saben por qué? Porque todas las luces se apagaron y los escenarios se cerraron para mí. Yo quisiera mostrar fuerza y belleza en la pantalla, pero me dan papeles en películas de mierda raras que nadie se interesa en ver (Sepúlveda y Adriazola, 2010).

En términos representacionales y para el tema del registro de la realidad que nos convoca particularmente en este estudio, es posible referir dos movimientos. En el primero, nos encontramos con puestas en obra que exprimen la operación de registro sobre la realidad, sosteniendo fragmentos indeterminados en pantalla y haciendo emerger, bajo un procedimiento intenso de auscultación y disección, la propia operación de registro sobre el mundo -como en el caso de Ningún lugar en ninguna parte (Torres Leiva, 2005) o de Noticias (Perut y Osnovikoff, 2009)-. En el segundo movimiento, 
nos encontramos con realizaciones que se arriesgan a la neo ficción, o ficción reformulada, trabajando en la frontera de la dualidad y relatividad entre documentalidad y dramatización, como ocurre en Rabia; El pejesapo; 31 de Abril, Manuel de Ribera o en El año del tigre (Lelio, 2011). En neo ficción, referimos asimismo el film Naomi Campbel, cuyo método de creación y realización incorpora a la propia Yermén protagonista - una joven transexual que anhela un cambio de sexo-, en el proceso de investigación y escritura del guion de ficción de una joven transexual que anhela un cambio de sexo y asiste a un casting que convoca un programa de televisión de cirugías plásticas. Yermén representa a Yermén en el film, se interpreta a sí misma, en su realidad, en un argumento que entrelaza realidad y ficción. La puesta en forma final del film incorpora grabaciones del tipo "caseras" que Yermén realiza en su población y de su propio día a día cotidiano durante la etapa de investigación que lleva a cabo con los directores. Lo documental se infiltra en la ficción, o la ficción se construye sobre el documental. Señalan los directores:

hay que dejar de mirar la vida desde un paradigma binominal; mujer/hombre, blanco/negro, realidad/ficción... El filme no aclara ni lo documental ni lo ficcionado acorde al proceso identitario: híbrido entre lo natural y la propia construcción. El cuerpo es un límite variable y permeable, y el cine por su parte no se puede considerar nunca una verdad pura, siempre hay un punto de vista y una cámara de por medio (telam.com.ar, web).

En esta línea, estamos ante un cine que trabaja, conscientemente, la relativización de las categorías preconcebidas de sentido narrativo asumidas por la convención.

Sobre estas distinciones, centramos a continuación la atención en la estrategia de registro que este cine Generación 2000 utiliza para narrar y poner en obra lo que se narra. En ello, la operación visual que recae sobre el encuadre presenta un quiebre -un choque, si se prefiere- determinante; punto de inflexión crucial en lo que hemos denominado cuadro-teatral y encuadre-cinematográfico para estos dos tiempos de la cinematografía nacional que revisamos en este trabajo. En términos operativoformales, distinguimos así dos frentes, no excluyentes y complementarios entre sí: el movimiento externo, asociado al tratamiento de cámara; y el movimiento interno, asociado al paisaje y a los personajes en cuadro.

Este cine opera muchas veces cámara en mano, con registros in situ captando en movimiento el devenir en movimiento; en ello, suprime distancias de observación y encuadres estables relevando espasmódicamente los cuadros y ángulos de mirada -como en el caso de El pejesapo y Rabia-; o bien, opera con encuadres deliberadamente estables, extendiendo el tiempo de observación sobre el cuadro fijo a esperas indeterminadas - como en los filmes Noticias y Manuel de Ribera-. En el primer caso, emerge la urgencia y el vértigo del tiempo real. Un sistema representacional que extrema la mirada en la inmediatez de lo circunstancial. La movilidad de la cámara -con su consecuente inestabilidad-, así como los planos y ángulos que se precipitan 
y ajustan con premura sobre las zonas de registro, ponen en forma los intersticios, los detalles, los accidentes, la fragmentación; subrayando la omnipotencia del tiempo real por sobre el tiempo narrativo ${ }^{19}$. En el segundo caso, asistimos a un tiempo que se sostiene en la espera y en la indeterminación. Es el peso del tiempo operando sobre el paisaje en la neutralización de la acción productiva. Acción debilitada en la desmesura de la dilatación temporal. El mundo: incierto, puro devenir. Emergencia del tiempo real. Y en ambos casos, ya sea por la visión compulsiva que opera sobre el paisaje o por la dilatación plástica del tiempo de auscultación, nos encontramos ante un tipo de registro y de representación de mundo que presenta el desfondamiento del sentido de la acción.

A modo de referencia:

En El pejesapo asistimos a la operación representacional de una poética del malestar. Y es que, por sobre el malestar argumental -el suicidio fallido que abre el film- es el malestar mismo de la propia representación el que pasa a primer plano en la misma representación. La mirada sobre el mundo que convoca el film es constantemente espoleada por la ambigüedad entre documentalidad y dramatización, así como por la fragmentación temporal, espacial y de parlamento. Por su parte, el desenfoque constante y las tomas a contraluz o en situaciones de escasa iluminación incorporan un grado significativo de indeterminación representacional. Para el final, el tiempo sigue transcurriendo y devuelve la imagen representacional de una impotencia fundamental. Para el final asistimos no al cumplimiento de sentido, sino a la interrupción de los esfuerzos del sujeto y a la interrogación.

En Rabia, la protagonista que busca trabajo y a la cual seguimos por varios días y en distintos lugares, todos relacionados con sus entrevistas de trabajo, deambula sumida en la extrañeza, en el sinsentido circundante, en la interrogación. La cámara en mano de un observador anónimo se concentra en la chica. Se instala a su lado, al frente de la fila que hace junto a otras postulantes para una entrevista más, o bien en la sala de espera de una oficina cualquiera, una como tantas, como si la cámara fuese, a ratos, una postulante más del grupo en el que se aguarda para ser llamado individualmente. Así las cosas, asistimos a un tiempo extendido en la acción debilitada, en el silencio de la chica, en la acción suspendida en la espera, en el plano sostenido de manera inestable que se vuelve motivo de auscultación e interrogación. Un tiempo que se plastifica en la suspensión. Un tiempo que, pese a transcurrir, se deshilvana en la indeterminación. Los personajes se hallan sometidos al paso del tiempo, en la

19 Entendiendo el tiempo narrativo como una operación de sentido que recae sobre el tiempo real, y que aplaca la inestabilidad originaria del sujeto. El tiempo narrativo operando sobre el tiempo real adopta la forma del "estar siendo en torno a", o bien, "en vías de". Y la espera, por su parte, condición inherente al ser, adopta la forma de un "todavía no"; situación en donde la espera, para el sujeto, asume la condición de encontrarse "en vías de" lograr el cumplimiento de lo que se espera. Todo gira en torno al sentido. A la narración como inscripción y como posibilidad de sentido. Es así que el tiempo narrativo, operando sobre el tiempo real, instala un presente con sentido y densidad; sistema que en este cine Generación 2000, distinguimos, está siendo alterado por la irrupción del tiempo real, por la cámara y sus operaciones sobre la realidad. 
espera caída de todo proyecto significativo; adoptando, el tiempo real, primacía por sobre el tiempo narrativo. Para el final, Rabia queda vuelta de cara a la frustración y el tiempo queda vuelto de cara a la profundidad.

En Manuel de Ribera, el protagonista toma contacto en distintas secuencias con lugareños de la isla mayor, les habla de su proyecto de fundación de la isla pequeña que ahora le pertenece, intentando convencerlos para que se vayan junto a él a poblar éste, su territorio. En el transcurso, los lugareños hablan como suelen hablar. Un habla exigua, que otras veces parece desvariar en sus propias inquietudes, ajena a las preguntas formuladas. A veces, en la isla, también se habla con silencios prolongados, a la espera de quién sabe qué, de algo que, con todo, no termina nunca de llegar. Un sentimiento de intemperie atraviesa todo el film. Es la isla, es la lejanía, es el paisaje, es la soledad del sujeto. No se trata sólo de la realidad, o de la ficción operando sobre la realidad, si no de una experiencia sobre la realidad: el desamparo y la orfandad, donde los esfuerzos del sujeto se vuelven, al final del día, al final del film, infructuosos y donde lo que insiste en la puesta en escena es la no pertenencia y la precariedad del sujeto. La imposibilidad, de ser, de pertenecer. En términos operativos, se trabaja con planos fijos y largos, silencios extensos, tiempos dilatados; lo que promueve una lectura de extrañamiento sobre el mundo circundante. El protagonista se encuentra, de algún modo, arrojado sobre la tierra de la isla y arrojado en el film.

El film Noticias articula fragmentos documentales de diversa índole y ambigua conexión: secuencias de monos en el zoológico, hospitales públicos con pacientes agonizantes en los corredores, reporteros de provincia, cadáveres humanos inventariados por la brigada policial, turistas en un salar desértico y procesiones religiosas populares con penitentes flagelantes. Parcelas de observación disímiles, tratadas preferentemente en planos exhaustivos, de cercanía extrema y descentramiento deliberado del posible foco de reconocimiento inmediato de la acción. El tiempo de registro, en tanto, dilatado sobre cada secuencia devuelve un tiempo suspendido en la observación e indeterminación. Sumado a lo anterior, el documental prescinde de una voz que guíe la lectura tanto de cada fragmento como de la articulación relacional entre ellos. En consecuencia, la abolición de la voz tutorial neutraliza el sentido narrativo haciendo emerger parcelas de mundo en una suerte de cirugía óptica y cirugía representacional. Así las cosas, el rendimiento crítico podemos situarlo en la operación de auscultación y, ciertamente, en la interrogación. Surge la pregunta a cerca del propósito de tal choque óptico, que nos conduce, a su vez, a la interrogación tanto del sentido de los recortes registrados como del sentido de la propia operación de registro y reproducción.

En Ningún lugar en ninguna parte, cuya bajada de título es: apuntes de un documental sobre la ficción, nos encontramos ante una suerte de cartografía visual que nos traslada al barrio La Matriz de Valparaíso. El film abre con La llegada del tren a la estación de los hermanos Lumière. Una vista silente y una referencia explícita a lo que en algún momento fue el incipiente registro de la realidad. En pantalla, un tren 
que llega a la estación de aquel entonces; actualidad que se desintegra en el propio registro y cristaliza, a estas horas, en memoria visual y reactivación de sentido narrativo. Un viaje que termina: el de la estación de 1895 y un viaje que se inicia: el de ningún lugar en ninguna parte. El viaje será, cierto, hacia La Matriz, pero el lugar específico funciona, a la vez, como una matriz medular que tiene la facultad de ser centro y propulsor. Centro de observación excepcional y propulsor de una operación de observación también excepcional, en la intención de ver -quizá- para acercar, para re conocer e interrogar. En ello, Ningún lugar en ninguna parte funciona a la manera de una esfera de múltiples cristales-espejo, que concentran infinitas texturas, materiales y humanas, fijas y móviles. Una fijeza que es transmutada en movilidad por obra del propio registro, de la cámara que se pone en movimiento; y movilidad transformada en fijeza por obra del plano fijo, del registro sostenido en la observación. Ningún lugar... equivale a un repertorio de innumerables puntos de contemplación, articulados -cada tanto- por la escena fragmentaria de dos chicas que ensayan una partitura musical en violín y violoncelo, y con las cuales - de hecho- la metáfora del viaje se inicia. Es el paisaje de la actual estación el que ahora se deja atrás: la gente, la construcción, los muros y los rieles. Para entonces, el plano en movimiento opera como contrapunto de la fijeza. Lo definido se vuelve textura óptica, evocación visual-táctil, tejido cromático vertiginoso y fugaz. Imagen vector articulada en el trayecto con planos fijos que recaen sobre muros, empedrado de calles, rostros anónimos que posan individualmente a cámara -literalmente, a la manera de una foto, presentándose casi inmóviles ante el registro y dispuestos a ser registrados-, el mar, la carretera solitaria, árboles elevándose hacia el cielo, nubes, en fin. Para estos recortes, la mirada se sostiene en un tiempo suspendido en la inacción. Es el tiempo operando sobre el paisaje, donde la acción productiva, o la hegemonía de la acción en un discurso narrativo clásico ha sido subvertida por la exposición al tiempo de observación. La sinopsis indica: "Es un recorrido personal a través de la imagen y el tiempo. Es el encuentro entre la realidad y la ficción. Es la constante búsqueda por encontrar una verdad dentro del documental. Es un ensayo musical. Es la constante preparación de un documental...” Ensayo, sinfonía y exploración documental. Recortes de el mundo, cartografía audiovisual que resplandece en estos apuntes de un documental sobre la ficción ${ }^{20}$.

En cuanto al movimiento interno, asociado a la composición del paisaje y a los personajes en cuadro; ya hemos introducido algunas señas. Tanto en el registro precipitado y urgente como en el de plano sostenido que somete la operación de observación a la espera, estamos ante un tiempo que se está deshilvanando en el transcurso ya sea de lo intrascendente, ya sea de lo indeterminado. Estamos, así, ante un cine particularmente incómodo, que hace emerger la operación de registro

20 Ficción que emerge en este documental por vía de la operación de sentido narrativo que recae sobre aquellos recortes de mundo dispuestos para la interrogación. 
sobre la realidad y la operación de observación, un cine que no propicia el olvido de la cámara y sus operaciones sobre la realidad. Emerge, en otras palabras, tanto para el realizador como luego para el espectador, la consciencia sobre la operación material de representación de mundo.

Con lo anterior, independiente del tema particular que cada film aborda, aquella composición teatral y el despliegue de la acción narrativa centrada en el rostro-masa, rostro-multitud, que distinguíamos en el cine silente, adopta aquí una condición inversa. El movimiento y la acción productiva, incluso el mismo sujeto, parecieran haber sido expulsados del cuadro. Lo que queda: puro devenir, indeterminación. En este sentido, en el cine Generación 2000 nos encontramos ante una puesta en forma crítica que nos traslada al plano de la interrogación tanto del sujeto como de la representación y posible aprehensión de mundo.

\section{Notas finales}

Trabajando en estos dos tiempos de la cinematografía nacional, la reflexión inicial nos llevó a identificar dos nociones preliminares que han guiado el análisis: el cuadroteatral y el encuadre-cinematográfico, la primera referida al cine silente y la segunda al cine contemporáneo. Tal distinción es -nos damos cuenta- arbitraria y no ha sido convenientemente explicitada. ¿En qué momento el cuadro se convierte en encuadre? ¿En qué momento lo teatral deja de ser tal y se convierte en cinematográfico? Si el cuadro de los comienzos es un cuadro en movimiento, una vista en movimiento, ¿no constituye con ello ya, acaso, un encuadre en términos cinematográficos? En fin, las preguntas sobre esta arbitrariedad nuestra pueden extenderse. Con todo, lo que hemos querido abordar y proponer con ello es aquel salto categórico en la operación visual que recae sobre el recorte y representación de mundo en estas dos épocas.

Cuadro y encuadre se hallan, evidentemente, ligados. Pero digamos, para clarificar, que el cuadro-encuadre (en esta secuencia, partiendo por "cuadro"), lo hemos situado en el cine de los comienzos, en el que hemos reconocido prima una disposición teatral del mundo en acto que se dispone a ser registrado y reproducido. El encuadre cinematográfico, en tanto, lo hemos entendido para el análisis como aquello que entra a escena toda vez que reconocemos en la poética artística la emergencia de una reflexión del tiempo y de los recursos materiales que participan en la aprehensión estética de mundo. Sin desmerecer ni los inicios, ni el transcurso de la historia de la cinematografía nacional; tal distinción la hemos situado en el cine Generación 2000, reconociendo que éste trabaja, particularmente, la exploración de las operaciones materiales, a la par de una búsqueda del cine como representación de mundo.

Con el advenimiento del Cinematógrafo, el cine de los comienzos se instala como una operación visual sobre el mundo y, en tanto cual, como discurso y representación. Estética de la toma de vistas -dirá Aumont-, que afirma la representación 
como operación sobre lo real (El ojo interminable 28). En esta operación visual, la naciente tecnología cinematográfica introduce un fenómeno sin precedentes en la meditación sobre la imagen representacional de un mundo en acto que, a través de la prótesis mecánica de visión instaura, ya preliminarmente, la potencia del tiempo de auscultación que proporciona el registro técnico. Potencia que, en sus inicios y como hemos revisado, se concentra en el espectáculo de la acción proferida y convocada por personas-personaje al interior del cuadro. Para entonces entra a escena el espectador del mundo cotidiano, en donde este acontecer "cotidiano" queda particularmente ligado a actualidades cívicas y sociales de la época.

Con lo anterior, en las primeras realizaciones chilenas -ya sea por imitación del modelo francés, ya sea por la influencia de la fotografía, ya sea por una técnica y lenguaje en exploración-, el encuadre cinematográfico actúa teatralmente y con una fuerza centrípeta, atrayendo a los sujetos de registro al cuadro, y en donde el peso de la representación de mundo recae en el peso que adopta la acción de los sujetos de registro en el mismo cuadro; registro de la actualidad cotidiana en el que las personas-personaje se vuelven, producto de la técnica, motivo de reproducción y de auscultación. Sobre lo mismo, hemos revisado que la "zona de parlamento" que convoca el movimiento de congregación al interior del cuadro, así como el plano sostenido sobre la multitud, actúa como un plano-masa, en donde la imagen que devuelve el lienzo de la pantalla queda particularmente ligada no sólo al rostro humano, si no que al rostro-masa. En ello, el cine silente pone en forma el rostro de la multitud, registrado en actualidades y acontecimientos nacionales. Es el despliegue de la acción de cara a la modernidad, al progreso, a la trascendencia.

En el cine contemporáneo Generación 2000, en tanto, la operación visual sobre el mundo adopta un rendimiento crítico vía la emergencia de las operaciones materiales y de la reflexión artística de los distintos niveles de sentido cifrados en la temporalidad. Tiempo precipitado y compulsivo, o bien, extendido y plastificado. Un tiempo, decíamos, que se está deshilvanando en el transcurso ya sea de lo intrascendente, ya sea de lo indeterminado.

Junto a lo anterior, es posible distinguir la fuerza centrífuga que ha adoptado con el tiempo aquel cuadro-encuadre teatral-cinematográfico de los inicios. Ya sea expulsando al sujeto de la zona de registro, o sosteniéndolo suspendido en la noacción, el peso de la representación de mundo recae en el peso del tiempo operando sobre el paisaje y sobre el mismo sujeto sumido en él; imagen de mundo que ha sido vaciada por algunos instantes de personas-personajes o que ha neutralizado la acción productiva, extendiendo el sentido de la temporalidad encapsulada a la intromisión de lo indefinido. En ello, el cine Generación 2000 pone en forma el desfondamiento del sentido de la acción y la imagen que devuelve el lienzo de la pantalla queda particularmente ligada al advenimiento de la indeterminabilidad, característica fundamental de nuestra época contemporánea. 
En cuanto a las nociones de fuerza centrípeta y fuerza centrífuga -la primera, operando sobre el cuadro del cine silente y la segunda sobre el encuadre del cine contemporáneo-, éstas se vinculan adyacentemente a lo propuesto por André Bazin en su momento a propósito del espacio en la escena teatral y del espacio en la pantalla cinematográfica (¿Qué es el cine? 183). En Bazin, el espacio centrípeto corresponde al del teatro, que atrae y concentra la acción en el centro de visión y exposición de la propia escena teatral. El espacio centrífugo, en tanto, corresponde al del cine, en donde la tensión constante entre campo y fuera de campo abre y proyecta los límites de lo que cada vez, reactualizándose, se halla en el campo de visión. En nuestra lectura, hemos desplazado ambos conceptos bazinianos a la representación cinematográfica, reconociendo la disposición teatral del cine de los comienzos y asociándolo a la fuerza centrípeta que actúa para entonces sobre la composición. Sobre ello, lo centrífugo lo hemos ubicado no sólo en el cine contemporáneo, sino en el propio encuadre del cine contemporáneo. Ya no campo y fuera de campo, en nuestra lectura nos detenemos en la composición de encuadre, de recorte de mundo, de representación; en aquello que permanece -en nuestros días - en el campo de visión: movimiento dominado por el desfondamiento del sentido de la acción. Atracción versus expulsión del cuadroencuadre teatral-cinematográfico.

Por su parte, el trabajo de Carolina Urrutia (Un cine centrífugo) también propone el concepto de centrífugo, en su caso, para las ficciones chilenas contemporáneas, centrando la atención en el cine comprendido entre los años 2005 y $2010^{21}$. Algunos de los filmes que aborda en el análisis coinciden con los nuestros, dado que su trabajo al ingresar al campo de estudio desde la ficción alcanza a aquellas realizaciones que nosotros hemos considerado como neo ficción o bien, a aquellas que en nuestro estudio hemos abordado desde la ruptura con el modelo narrativo-técnico convencional y que complejizan particularmente el tema de la mirada en y sobre la representación de mundo. El estudio de Urrutia enriquece la lectura que hemos abordado de filmes como El pejesapo, Manuel de Ribera, Mitómana o Rabia. Pero, por sobre todo, alienta reconocer que su trabajo se alinea con nuestro pensamiento a cerca del cine chileno Generación 2000. Señala que no estamos solos, que quienes pensamos el cine lo estamos interrogando desde el mismo frente y reconocemos que una parcela importante de nuestro cine "destierra ciertos presupuestos narrativos, argumentales y estéticos que instituyó el cine chileno de los años noventa, para instalarse - en la actualidad- en territorios nuevos y, a nuestro parecer, poética, estética y lingüísticamente, enriquecidos" (13).

Sobre lo anterior, Urrutia a su vez comprende lo centrífugo como:

un movimiento que opera como contrapunto de un movimiento centrípeto y que, en el cine, se manifestaría a partir de narraciones que tensan las relaciones

21 El libro de Carolina Urrutia se publica en el 2013. Para entonces, nuestro estudio en fase final recoge su análisis reconociendo que enriquece la lectura que habíamos seguido en este trabajo. 
entre lo dinámico y lo estático, que reflexionan sobre las velocidades y lentitudes de los cuerpos que habitan un plano, sobre los paisajes que se despliegan en un plano (Ibíd.).

Distingue que en estas narraciones el espacio se vuelve protagónico, "se fuga la figura del pueblo, de la masa (que sale de cuadro) y lo que queda es el paisaje” (16). En el movimiento centrífugo, señala Urrutia, "algo se expulsa, el acontecimiento (el gran acontecimiento, el suceso edificante) se desplaza fuera de campo, la historia se deshilvana y en el centro queda el sujeto" (125). Entre ficciones, neo ficciones y documentales, finalmente lo que prevalece es la pregunta por la representación.

Como hemos revisado en el trayecto de este escrito, nuestro análisis ha trabajado las nociones de centrípeto y centrífugo para distinguir la manifestación representacional que opera sobre el cine silente y el cine Generación 2000, ensayando una lectura de desplazamiento del cuadro teatral al encuadre cinematográfico. Así las cosas, el cambio narrativo-técnico que hemos reconocido entre estos dos tiempos de la cinematografía nacional no radica sólo en la manera de registrar y producir, o en las modificaciones técnicas sufridas por el propio mecanismo de captación de escenas, sino en la búsqueda artística del cine como representación de mundo, como posibilidad de comprensión e inscripción del sujeto. En esta vía, el efecto de realidad del cine de los comienzos se vuelve, en el cine contemporáneo, un efecto de pensamiento sobre lo real. Sobre el registro y reproducción de escenas de actualidad, cuestión que en el cine silente resguarda el imaginario moderno vuelto hacia la confianza en el progreso; se levanta, en el cine Generación 2000, la reflexión sobre los procedimientos técnico-narrativos implicados en la operación de registro y aprehensión de mundo. Un mundo que, de cara a la contemporaneidad, recordando las palabras de Paul Virilio: "no termina nunca de llegar, que no acabamos nunca de esperar" (La estética de la desaparición 66).

El interés de presentar esta lectura de la cinematografía nacional, ha sido establecer coordenadas de navegación que contribuyan a la discusión respecto de la representación y el estatuto de realidad en el cine de lo real. Sobre lo mismo, consideramos que, en una siguiente etapa, sería apropiado interrogar desde la particularidad del cine chileno y en relación con el cine latinoamericano, aquello que emerge de propio, tanto en el cine silente como en el cine contemporáneo nacional, distinguiendo lo que se aproxima en ello en términos socio-culturales y aquello que se releva. 


\section{Referencias}

Aumont, Jacques. El ojo interminable. Barcelona: Editorial Paidós, 1988. Medio impreso. Bazin, André. ¿Qué es el cine?. Madrid: Ediciones Rialp. 1990. Medio impreso.

Benjamin, Walter. Discursos interrumpidos I. Madrid: Taurus Ediciones, 1973. Medio impreso.

Campo, Javier. "Los estudios de cine documental y la cuestión de lo real". Comunicación y Medios, 24. (2011): 273-284. www.comunicacionymedios.uchile.cl. Fecha de ingreso: 29 de junio 2012. Sitio web.

Canal elbuentiempo. "Haciendo Manuel de Ribera" (2010). www.youtube.com/ watch?v=C5_j-3h2k40. Fecha de ingreso: 19 de diciembre 2010. Sitio web.

Canal onoff. "Brainstorming Iván Osnovikoff” (2011). www.youtube.com/ watch?v=70udaWCRoBE. Fecha de ingreso: 3 de marzo 2012. Sitio web.

Chile Independiente. "Entrevistas. José Luis Sepúlveda, realizador de El Pejesapo". (s.f.). www.chileindependiente.cl/entrevista_jlsepulveda.php. Fecha de ingreso: 31 de agosto 2011. Sitio web.

Doane, Mary Ann. La emergencia del tiempo cinemático. La modernidad, la contingencia y el archivo. Murcia: Cendeac. 2012. Medio impreso.

Flores, Carlos. Excéntricos y Astutos. Influencia de la conciencia y uso progresivo de operaciones materiales en la calidad de cuatro películas chilenas realizadas entre 2001 y 2006. Santiago: Colección Cine, Programa de Magíster en Teoría e Historia del Arte, Universidad de Chile, 2007. Medio impreso.

Griffero, Ramón. La dramaturgia del espacio. Santiago: Frontera Sur - Edicionesartesdelsur. 2011. Medio impreso.

Godoy, Mario. Historia del cine chileno. Santiago: Imprenta Fantasía, 1966. Medio impreso.

Jara, Eliana. Cine mudo chileno. Santiago: Fondo de Desarrollo de la Cultura y las Artes, 1994. Medio impreso.

---. "Una breve mirada al cine mudo chileno con sus aciertos y descréditos". Revista de Letras 46. (2010): 175-191. Medio impreso.

Parada, Marcela. "Los estudios sobre cine en Chile, una visión panorámica. 19602009”. Razón y Palabra 77. (2011). www.razonypalabra.org.mx/varia/77\%20 5a\%20parte/67_Parada_V77.pdf. Fecha de ingreso: 4 de febrero 2015. Sitio web.

Telam.com.ar. "Llega 'Naomí Campbell', largometraje chileno sobre una travesti que busca cambiar de sexo" (2015). www.telam.com.ar/notas/201502/94021-lleganaomi-campbell-largometraje-chileno-sobre-una-travesti-que-busca-cambiarde-sexo. Fecha de ingreso: 2 de agosto 2015. Sitio web.

Urrutia, Carolina. Un cine centrífugo. Ficciones chilenas 2005-2010. Santiago: Editorial Cuarto Propio. 2013. Medio impreso. 
Vega, Alicia. Itinerario del cine documental chileno: 1900-1990. Santiago: Centro EAC, Ediciones Universidad Alberto Hurtado. 2006. Medio impreso.

Virilio, Paul. La estética de la desaparición. Barcelona; Editorial Anagrama, 1988. Medio impreso.

Zavala, Lauro. “Cine Clásico, Moderno y Posmoderno”. Razón y Palabra 46 (2005). www.razonypalabra.org.mx/anteriores/n46/lzavala. Fecha de ingreso: 25 de julio 2011. Sitio web.

Recibido: 23 septiembre 2013 Aceptado: 22 junio 2015 